oxygen, etc. Ether is admitted to the apparatus in one of two ways-by a Clover inhaler, or by the oxygen tube from a Boyle's machine.

\section{Technigue of Administration}

The canister is supported by clips to the Boyle's machine, leaving the face-piece free. The patient is induced with gas and oxygen till consciousness is lost. Then a Clausen harness is fastened over the face to ensure a gas-tight fit of the face-piece: this is most important. The expiratory valve is now closed down tightly and the gas flow stopped. The oxygen supply is reduced to 300 to 500 c.cm. per minute. Ether is now added from the Clover if this instrument is in use, or by the oxygen being allowed to bubble through the ether in the Boyle's machine.

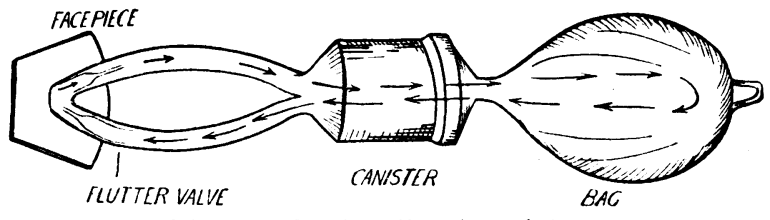

Diagram showing direction of flow.

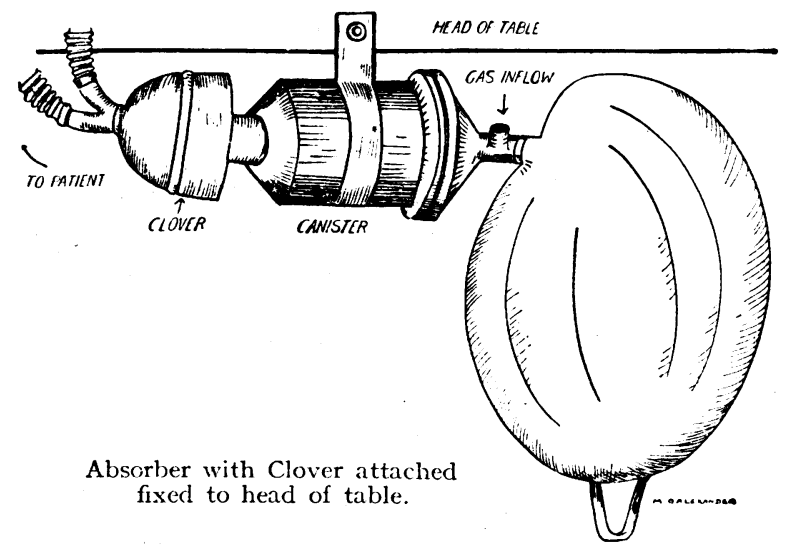

In practice it is found that some nitrous oxide always leaks away, so that a few cubic centimeters are added from time to time to keep the bag full, but actually only one to two gallons are needed per hour. One cannot use this method on less, as gas always leaks away a little, and it has been shown that nitrous oxide is excreted by the skin in small amounts. One charge of soda lime lasts for six to eight anaesthetic hours and all the apparatus can be sterilized by boiling. The flutter valves are easily replaced, and give no trouble. No provision has been made for switching out the absorber from the circuit. It is so easy to plug the bag directly. on to the Y-piece that it was felt advisable to avoid further sources of possible leaks. In practice it is found that the respiration rate with the absorber in use is quite unchanged, its depth, too, being almost unaltered, and not nearly as great as that obtained with a Clover's ether inhaler or similar closed method of anaesthesia.

\section{Conclusions}

The method has no disadvantages. Its advantages, which are many, are as follows:

1. To the patient: (a) No loss of heat through the lung, and therefore $(b)$ less risk of bronchitis, etc. (c) Minimal use of ether.

2. To the anaesthetist: (a) No smell of anaesthetic in the theatre. (b) Economy in gas and ether. (c) Extreme portability - twenty-five-gallon cylinders need only be carried instead of hundred-gallon ones-a great saving in weight.

The apparatus was made for me by A. Charles King, Ltd., Devonshire Street, W.1.

\section{THE USE OF TAKAYAMA'S SOLUTION IN THE IDENTIFICATION OF BLOOD STAINS * \\ BY}

\author{
AUBREY V. GREAVES, M.B.TOR.
}

M.C.P. AND S.ONT., D.T.M.LJVERP.

GOVERNMENT BACTERIOLOGIST, HONG-KONG ; LECTURER IN FORENSIC MEDICINE AND TOXICOLOGY, UNIVERSITY OF HONG-KONG

Though the importance of the production and identification of crystals derived from blood in medico-legal cases has received much emphasis in the literature, little change has occurred in recent years in the methods employed for their demonstration.

\section{History OF TESTS}

Teichmann in 1853 noted the appearance of brownish rhombic crystals when blood was treated with glacial acetic acid and a trace of sodium chloride. These crystals were later identified as being those of haematin chloricle, and since then it has been recognized universally that their presence is proof of the identity of the suspected stain with blood. Unfortunately it was soon found that such crystals could only be demonstrated with difficulty and uncertainty. Many attempts to modify the technique and to simplify the procedure were made, but few of these modifications had much to offer. Probably the best was devised by Sutherland, ${ }^{1}$ who suggested that a small drop of normal saline should be evaporated to dryness on a slide before the fragments of the suspected stain were deposited on it; glacial acetic acid was then added and the slide heated gently. Even this method frequently fails of its object. Later Donogány and Bürker ${ }^{2}$ suggested the use of pyridine for demonstrating haemochromogen crystals in blood. Pyridine used alone, however, gave rather uncertain results, and attempts were made to devise a solution containing this reagent which would be more successful. Takayama ${ }^{3}$ in 1912 suggested a solution of the following composition :

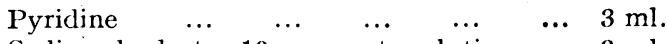

$$
\begin{aligned}
& \text { Sodium hydrate, } 10 \text { per cent. solution } \quad \ldots \quad 3 \mathrm{ml} \text {. } \\
& \text { Dextrose, sat. solution } \quad \ldots \quad \ldots \quad \text {... } 3 \mathrm{ml} \text {. }
\end{aligned}
$$

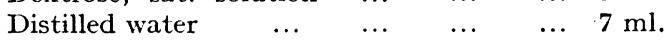

Mahler $^{4}$ in 1923, and Kerr and Mason ${ }^{5}$ in 1926, reported favourable results with this solution, and since this time it has found mention in a hesitating way in some of the textbooks, though it has obviously not attained the recognition to which its usefulness entitles it. It has given unfailing satisfaction in my own hands, and I feel that it deserves to be incorporated more widely into routine usage. Sutherland, in a textbook article on blood stains, describes his own routine method of examination of suspected stains as follows: He removes a tiny fragment of the material containing the stain, plunges it into boiling water for a few seconds, transfers it to a.slide, and removes the water by blotting. A drop or two of 10 per cent. $\mathrm{KCN}$ solution is now added, and the slide again blotted; finally, a drop of strong ammonium sulphide solution is placed on the fragment, a cover-slip adjusted, and the preparation examined microscopically. If blood is present a salmon-pink colour will be seen, and microspectroscopic examination will show the spectrum of haemochromogen. He feels that no further proof of the presence of blood is required.

\section{Technigue of Method}

The method of using Takayama's solution to be described accomplishes all that Sutherland's method does, but in addition demonstrates haemochromogen crystals,

* From the Bacteriological Institute, Medical Department, HongKong (Hon. Dr. A. R. Wellington, Director of Medical and Sanitary Services). 
and does so with simplicity and certainty. I have never failed to obtain crystals in a known positive case. Occasionally an old stain may require a little longer treatment, but the result seems to follow just as surely. It must be emphasized that the reagent should be freshly prepared if prompt reaction is expected. It gives satisfactory results for about two months only, although I have obtained crystals using a solution six months old.

The method is as follows. With a sharp scalpel a portion of the suspected stain is scraped off the weapon or other article on to a clean slide. The smaller the fragment the better. The largest portion on the slide should not exceed about half the size of an ordinary pin's head. I find that the minute scraping which sometimes adheres to the scalpel usually suffices; this often has to be removed with another scalpel or some instrument possessing an edge. Taking care that this is not blown off the slide, a drop of Takayama's solution is placed on it and a cover-slip adjusted. It is important that the drop be not too large, otherwise the cover-slip will " float " on the surface, and the formation of crystals will be delayedr In the case of a stain on fabric a good method of procedure is to cut out the stained portion, and having placed it on the centre of a slide, to scrape gently the surface of the stain with a sharp scalpel until a few minute fragments of " fluff" are deposited on the slide; a drop of the solution is then added as before. It is necessary that the laboratory bench be free from air draughts, lest the material on the slide be blown away before the solution is added. On microscopical examination (which should be immediate), if the blood is present a distinct pinkish area will very soon be seen among the debris on the slide. As soon as this is identified the ocular of the microscope should be removed and the tube of a hand spectroscope with a wave-length scale inserted in its place. The characteristic bands of haemochromogen should be made out and the reading of the scale taken. Emphasis is laid on the necessity for immediate examination, as I have found that the spectrum is much more distinct and more surely obtained before crystal formation than after. Usually, if the blocd stain is reasonably fresh, crystals will form in from two to five minutes, but it is well to leave a slide for re-examination some hours later before recording a negative.

The crystals are so characteristic in colour and shape that they cannot be mistaken by an observer with even a little experience. They are of a pink to salmon-pink colour, and in shape are flat and rhomboid. The ends of individual crystals are often splintered. Some crystals terminate in a blunt point like the blade of a pocketknife ; others, again, are quite rectangular. Seen on edge they are like sharp lines. There is a strong tendency to " sheaf" formation. The best crystals are the rather fine, separate ones seen off the edge of the fragment, actually in the solution, against a clear background. These take longer to form and are rather small, but are well worth study under the oil-immersion lens. Another characteristic type of crystal which I have seen occasionally is derived from fairly fresh blood stains. The crystals are curved instead of flat, and tend to lie so as to present their thin surfaces uppermost, the result being a number of fine, curved, dark pink lines, giving a most striking appearance to the microscopical field. Such crystals as do not lie completely on their sides may be seen to present the same rectangular structure, and there are practically always plenty of the more usual flat forms to be seen as well.

The colour change described by Kerr and Mason after the addition of Takayama's solution-namely, greenbrown, dark red to pink-has not been observed, certainly by myself, but this does not seem of great importance. I have found the use of heat entirely unnecessary; where delay occurs in crystal formation I merely leave the preparation at room temperature for fifteen minutes to half an hour, examining it at intervals. Should the material for study be scanty and crystals few and possibly very fine, necessitating the use of a high magnification, care should be taken to focus down very slowly and cautiously to watch for fine crystals on the surface of the preparation. Often this is the first place at which crystal formation occurs, and they may be picked up at this point many minutes before being seen at any other level in cases of difficulty. It is unfortunately very easy to focus past the surface and miss these crystals. Where they are slow in appearing I have found that they may at times be caused to appear by gently tapping the microscope stage.

\section{General Considerations}

Carried out in this manner we thus have a perfectly definite diagnostic triad: a colour reaction, the formation of specific crystals, and the production of certain bands at specific points in the spectrum. No other known substance but blood will give these three reactions in the circumstances described. If a positive result has been obtained in this manner no further test is necessary in order that testimony may be borne as to the presence of blood. In fact, it is bad practice to use other tests, the results of which may possibly be negative in the presence of blood and whose positivity does not alone justify one in affirming that the stain is of blood. Should such tests be negative or doubtful the positive evidence already obtained is immediately weakened in the eyes of a jury. Logically speaking, a test the results of which are open to criticism cannot strengthen the results of one possessing an absolute value. It is good practice, however, if sufficient material is available, to obtain a solution of the stain and to demonstrate, if possible, the spectrum of oxy-or methaemoglobin macroscopically and then by treatment with caustic soda and ammonium sulphide solution obtain the spectrum of haemochromogen. This, too, is also an absolute test for blood. Unhappily the material available is all too frequently insufficient for this procedure, and it is in such cases especially that the procedure described is invaluable.

No claim to originality in the use of the method is intended, but it is felt that a description of a good routine application of Takayama's solution is needed and that some such method deserves far wider usage than it appears to have at the present moment among those practising forensic medicine.

\section{References}

${ }^{1}$ Sutherland, W. D.: Blood Stains, Baillière, Tindall and Cox, 1907.

2 Bürker: Münch, med, Woch, 1909, S, 123.

3 Takayama: Japanese Journal of Toxicology, 1912.

"Mahler, K.: Deut. Zeit. f. d. Ges. Gerichtl. Med., 1923, ii, 671.

Kerr, J. A. K., and Mason, V. H.: British Medical Journal. January 23rd, 1926

\section{TWO FURTHER CASES OF SARCOMA ASSOCIATED WITH KNOWN TRAUMA BX}

DAVID H. HALER

pathologist, infants hosprtal, S.W. ; LATELY asSistant pathologist, PRINCE OF WALES'S HOSPITIL, N.

In a recent article in the British Medical Journal I was able to record two cases of sarcoma in association with a known trauma. In the following note $I$ propose to describe two similar cases, wherein two totally different organs were involved.

\section{CASE I}

In the first case the patient, a boy of 17 , was playing with his $\log$ in the garden when he received a blow on the right testicle. This was attended with marked pain and rapid swelling, which was treated as a traumatic haematocele. As 